\title{
Tailoring photonic forces on a magnetodielectric nanoparticle with a fluctuating optical source
}

\author{
Juan Miguel Auñón, ${ }^{1}$ Cheng Wei Qiu, ${ }^{2}$ and Manuel Nieto-Vesperinas ${ }^{1, *}$ \\ ${ }^{1}$ Instituto de Ciencia de Materiales de Madrid, Consejo Superior de Investigaciones Científicas Campus de Cantoblanco, \\ Madrid 28049, Spain \\ ${ }^{2}$ Department of Electrical and Computer Engineering, National University of Singapore, 4 Engineering Drive 3, Singapore 117576, Singapore
}

(Received 24 July 2013; published 10 October 2013)

\begin{abstract}
We address the forces exerted by the random electromagnetic field emitted by a fluctuating optical source on a kind of dielectric nanoparticles that have arisen much interest because of their recently shown magnetodielectric behavior. The illumination with light, or other electromagnetic wave, of a given state of coherence allows us to create photonic forces, a particular case of which are optical analogous to the Casimir-Polder and van der Waals forces, as well as of thermal forces out of thermodynamic equilibrium. This leads to a deeper understanding of the conditions and limitations under which some theories of these forces were established. We also study the effects of the coherence length and of sharp changes in the particle differential scattering cross section due to Kerker minimum forward or zero backward conditions. We show how the nanoparticle Mie resonances, constituted by the induced electric and magnetic dipoles, lead to long distance attractions to the source, as well as to the possible predominance of magnetic forces. In addition, it is shown how, by manipulating the fluctuating source, either pushing or tractor beams are obtained, even in the far zone. These effects are specially relevant when quasimonochromatic emission is employed, and manifest the possibility of performing a monitoring of these mechanical interactions, in particular by a photonic analogy of those aforementioned classical thermal forces. This opens paths to nanoparticle ensembling and manipulation. The influence of the excitation of surface waves of the source is also considered.
\end{abstract}

DOI: 10.1103/PhysRevA.88.043817

\section{INTRODUCTION}

Many previous studies have addressed the response of atoms and nanoparticles to random radiation forces, specifically those due to fields from a fluctuating source [1]. Most of them have dealt with $\delta$-correlated sources, such as those thermal and blackbodies, specially in connection with the Van der Waals (VdW) and Casimir-Polder (CP) interactions [2-17]. Recently, magnetodielectric particles, made of a nonmagnetic material of high permittivity such as ceramics in the microwaves and semiconductors in the optical regions, have attracted much attention due to their exotic properties as scatterers and nanoantennas, as a consequence of the coupling between their electric and magnetic dipoles induced by the illuminating light field [18-26]; in addition, they are excellent laboratory systems to test and tailor the effects of such interactions [18,19,27].

In this work we deal with a general kind of statistical source in a regime of wavelengths different to that of the thermal radiation studied in previous works. Namely, we consider those that emit at visible and near-infrared (NIR) frequencies, and that are both spatially partially coherent and of the wide variety known as statistically homogeneous and isotropic $[1,28]$. Their emission excites fluctuating electric and magnetic dipoles of the particle placed in its near field which, in turn, may be considered as secondary sources whose radiation interacts with the aforementioned source which we refer to as the primary one. This gives rise to a total optical force that results from the combined action of the fields radiated by both the primary statistical source and from those fluctuating dipoles.

At thermal wavelengths, the interaction from the nanoparticle induced dipoles is interpreted as a Liftshitz force [29],

\footnotetext{
*mnieto@icmm.csic.es
}

which in the limit of zero temperature $T$ becomes those from vacuum fluctuations, i.e., those derived by either $\mathrm{VdW}$ and $\mathrm{CP}$ [30], depending on the distance, and also on the use or not, of a quasistatic formulation.

Nevertheless, the optical frequencies $\omega$ addressed in the present study are such that $\hbar \omega / k T \gg 1$ at $T=300 \mathrm{~K}$, and hence Planck energy becomes the same as that of the vacuum fluctuations: $\hbar \omega\left\{\frac{1}{2}+1 /[\exp (\hbar \omega / k T)-1]\right\} \approx \frac{1}{2} \hbar \omega$. Hence, if we considered the emitting optical source spectrum as just given by a Planck distribution, the forces in the visible and NIR ranges due to the particle induced fluctuating dipoles will be the optical analogous to those from the vacuum fluctuations in the thermal spectrum, namely $\mathrm{CP}$ and VdW. Thus our optical system constitutes an excellent means to create, test, and monitor photonic analogous of such thermal forces as well as of those out of thermodynamic equilibrium [4,5].

However, due to the magnetic response of the nanoparticle, more forces come into play in addition to those from the excitation of the electric dipole of a conventional dielectric particle, and they keep the above-mentioned analogy with those of VdW, CP, and out of equilibrium interactions. Such additional forces come from the excitation of a magnetic dipole in the nanoparticle, thus allowing a larger number of degrees of freedom of relevance for the control of the mechanical interaction and hence for object ensembling and manipulation.

It should be stressed that since the total force conveys adding the contribution of waves emitted by the random source at all frequencies of its spectrum, the most interesting cases in the NIR and optical frequencies are those in which these fluctuating sources are quasimonochromatic $\left(\Delta \omega \ll \omega_{0}\right.$, with $\Delta \omega$ denoting the bandwidth) [1]. Then the behavior of the nanoparticle polarizability as the central frequency $\omega_{0}$ varies leads to a rich and most interesting landscape of optical forces. This is in contrast with what happens when the 
source emits with a broad spectrum such as that of a Planck distribution, a particular case of which are, e.g., those thermal widely considered in connection with $\mathrm{CP}$ and $\mathrm{VdW}$ forces. In this latter case such effects of the radiation force, if there existed a particle, or object, with such a rich response to the electric and/or magnetic vector of the emission in that range of frequencies, would be washed out.

In addition, it must be remarked that out from the Rayleigh and quasistatic approximations widely used so far for the study of thermal forces on atoms and particles, the obtention of the radiation force by frequency integration over a wide emission spectrum such as that given by Planck's law loses its significance because all Mie resonance lines of the nanoparticle would then be swamped by this wavelength superposition. In fact, the same happens also with the dispersion resonances, present as the poles of the particle permittivity $\varepsilon_{p}(\omega)$, as well as with those of the static plasmon: $\varepsilon_{p}=-2$.

Hence here we discuss the mechanical interaction of a small particle, generally being magnetodielectric, with the random fields from a fluctuating optical source whose general spectrum and bandwidth may be controlled at will: e.g., whether quasimonochromatic, or with a broader bandwidth, in particular one may deal with one given by a Planck-like distribution, and whose coherence length is also monitored. The nanoparticle has a rich spectral response to both the fluctuating electric and magnetic vectors and, thus, much beyond previous studies on $\mathrm{CP}$ and VdW thermal effects, a wealth of the force landscapes may be observed by controlling the constitutive and emissive parameters of the particle and the source, respectively.

A main message of this work is that while these secondary source (i.e., the particle induced dipoles) forces can become relatively negligible by conveniently manipulating the power of the primary source, we show configurations and experimental designs where they are made to predominate. Hence there is a choice by selecting the frequency, or the spectral position and bandwidth of illumination, to decide the predominance of the induced dipole fluctuations (optical analogies and generalizations of Casimir-Polder, Van der Waals, or out of thermodynamic equilibrium forces), or the mechanical dominance of the emission from the primary fluctuating source.

An additional consequence of this analysis is that it manifests the limitations of previous studies, and the way to remedy them, concerning the asymptotic spatial dependence of radiation forces. As we shall show, these shortcomings stem from the use of the quasistatic approximation out from its domain of validity, for either the random emitted field or for the particle response characterized by its polarizability.

\section{FORCES ON A MAGNETODIELECTRIC NANOPARTICLE FROM A PARTIALLY COHERENT RANDOM ELECTROMAGNETIC FIELD}

The geometry considered in this paper consists of two regions (cf. Fig. 1). The one in $z<0$ is occupied by the random source with its polarization currents and it will be denoted as 1 ; whereas the one in $z>0$, standing as 2 , is free space and contains the nanoparticle. The fluctuating source considered here may be any random emitting medium; for

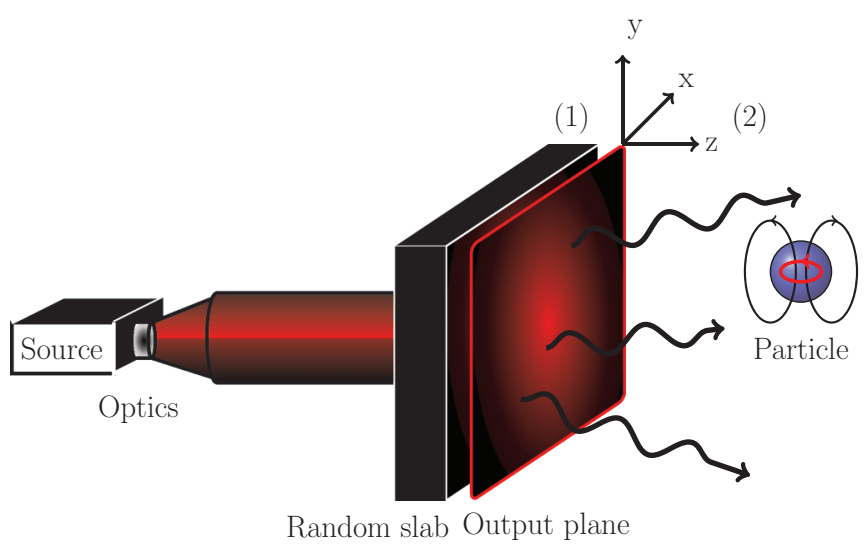

FIG. 1. (Color online) Scheme of setup and system geometry.

instance, for laboratory experiments it can be, e.g., a random refractive index, or a random rough surface dielectric slab illuminated by either quasimonochromatic radiation (like a laser), or by any other source such as a lamp of any chosen spectral line shape and bandwidth. Then, since this system is ergodic, on moving, e.g., rotating, the slab, ensemble averages, equivalent to time averages, are performed in such a way that the coherence length of the field emitted (i.e., transmitted) by this slab is defined by the correlation length of the random medium inhomogeneities (see, e.g. [31,32]).

\section{A. Forces from the primary source}

The Cartesian components of the ensemble-averaged force exerted by a random field on a magnetodielectric dipolar nanoparticle is the sum of an electric, magnetic, and electricmagnetic dipole interference parts [28,33,34], which are expressed in terms of the first electric and magnetic Mie coefficients $a_{1}$ and $b_{1}$, or corresponding polarizabilities $\alpha_{e}$ and $\alpha_{m}$, as [33]

$$
\begin{aligned}
\left\langle F_{i}(\mathbf{r})\right\rangle= & \left\langle F_{i}^{e}(\mathbf{r})\right\rangle+\left\langle F_{i}^{m}(\mathbf{r})\right\rangle+\left\langle F_{i}^{e m}(\mathbf{r})\right\rangle \\
= & \frac{\varepsilon_{0} \varepsilon_{2}}{2} \operatorname{Re}\left\{\left\langle\alpha_{e} E_{j}(\mathbf{r}) \partial_{i} E_{j}^{*}(\mathbf{r})\right\rangle\right\} \\
& +\frac{\mu_{0} \mu_{2}}{2} \operatorname{Re}\left\{\left\langle\alpha_{m} H_{j}(\mathbf{r}) \partial_{i} H_{j}^{*}(\mathbf{r})\right\rangle\right\} \\
& -\varepsilon_{0} \varepsilon_{2} \frac{Z k_{0}^{4}}{12 \pi} \operatorname{Re}\left\{\left(\alpha_{e}^{*} \alpha_{m}\right)\left\langle\mathbf{E}^{*} \times \mathbf{H}\right\rangle_{i}\right\},
\end{aligned}
$$

where the angular brackets denote ensemble average, $i, j=$ $1,2,3, \varepsilon_{l}=\varepsilon_{l}^{\prime}+i \varepsilon_{l}^{\prime \prime}$, and $\mu_{l}=\mu_{l}^{\prime}+i \mu_{l}^{\prime \prime}(l=1,2)$ are the permittivity and susceptibility of the medium embedding the particle, respectively, in our case being vacuum, and $Z=$ $\sqrt{\mu_{0} \mu_{2} / \varepsilon_{0} \varepsilon_{2}}$. This means that the small particle is considered as dipolar. However, it is large enough to require a Mie formulation with the first electric and magnetic partial waves fully describing its scattering. The electric and magnetic polarizabilities of the particle, $\alpha_{e}$ and $\alpha_{m}$, are then expressed as $\alpha_{e}=i \frac{3 \epsilon_{0}}{2 k_{0}^{3}} a_{1}$ and $\alpha_{m}=i \frac{3}{2 \mu_{0} k_{0}^{3}} b_{1} . E_{i}(\mathbf{r})$ is the total electric vector at frequency $\omega$ at any point of the half-space $z>0$; hence at the position of the particle, i.e., at $\mathbf{r}=\mathbf{r}_{0}$, it will be

$$
E_{i}\left(\mathbf{r}_{0}, \omega\right)=E_{i}^{\mathrm{inc}}\left(\mathbf{r}_{0}, \omega\right)+E_{i}^{p}\left(\mathbf{r}_{0}, \omega\right)+E_{i}^{m}\left(\mathbf{r}_{0}, \omega\right)
$$


The associated magnetic field $\mathbf{H}$ can be directly obtained from Maxwell's equations [35]. Whereas in Eq. (2) the first term represents the electric field emitted from the primary source constituted by the random slab and incident on the nanoparticle, the last two terms are the electric fields emitted by the particle induced dipoles: $p_{i}=\varepsilon_{0} \alpha_{e} E_{i}^{\text {inc }}$ and $m_{i}=\alpha_{m} H_{i}^{\text {inc }}$, respectively, after multiple reflections at the plane $z=0$ [36]

The electric-field incident on the particle is defined through the Green's function $G_{i j}^{E P}$, which includes the transmission Fresnel coefficients $t_{s, p}$ from $z<0$ into $z>0 . G_{i j}^{E P}$ may be written as a superposition of plane waves. Thus, in terms of the polarization currents, one has

$$
E_{i}^{\mathrm{inc}}\left(\mathbf{r}_{0}\right)=\mu_{0} \omega^{2} \int_{V} G_{i j}^{E P}\left(\mathbf{r}_{0}, \mathbf{r}^{\prime}, \omega\right) P_{j}\left(\mathbf{r}^{\prime}, \omega\right) d^{3} r^{\prime} .
$$

$V$ denotes the volume occupied by the source. A more detailed description about these Green's functions can be found in, e.g. $[37,38]$ and in the Appendixes. Note that, for $p$ polarization, $t_{p}$ support surface plasmon polaritons (SPPs) when $\varepsilon_{1}^{\prime}<1$.

Now we define the cross-spectral density tensor of the source polarization $P_{i}(\mathbf{r})$ as $W_{i j}^{(P)}\left(\mathbf{r}_{1}, \mathbf{r}_{2}, \omega\right)=$ $\left\langle P_{i}^{*}\left(\mathbf{r}_{1}, \omega\right) P_{j}\left(\mathbf{r}_{2}, \omega\right)\right\rangle$. We shall address the wide variety of statistically homogeneous and isotropic sources [1] for which

$$
W_{i j}^{(P)}\left(\mathbf{r}_{1}, \mathbf{r}_{2}, \omega\right)=S^{(P)}(\omega) \mu_{i j}^{(P)}\left(\left|\mathbf{r}_{1}-\mathbf{r}_{2}\right|, \omega\right) .
$$

$S^{P}(\omega)$ denotes the power spectrum of the source and $\mu_{i j}^{(P)}\left(\left|\mathbf{r}_{1}-\mathbf{r}_{2}\right|, \omega\right)$ is the spectral degree of coherence [1]. We assume a Gaussian degree of coherence; therefore, the correlation function reads $W_{i j}^{(P)}\left(\mathbf{r}_{1}, \mathbf{r}_{2}, \omega\right)=\int^{(P)}(\omega) \exp \left[-\left(\mid \mathbf{r}_{1}-\right.\right.$ $\left.\left.\mathbf{r}_{2} \mid\right)^{2} / 2 \sigma^{2}\right] \delta_{i j} /(2 \pi)^{3 / 2} \sigma^{3}, \sigma$ being the coherence length of the source and $S^{(P)}(\omega)=\int^{(P)}(\omega) /(2 \pi)^{3 / 2} \sigma^{3}$ representing the normalized spectrum.

A special case of these sources are those thermal and blackbodies widely studied, for which $\sigma \rightarrow 0$, so that $W_{i j}^{(P)}\left(\mathbf{r}_{1}, \mathbf{r}_{2}, \omega\right)=\int^{(P)}(\omega) \delta\left(\left|\mathbf{r}_{1}-\mathbf{r}_{2}\right|\right) \delta_{i j}$ and $\mu_{i j}^{(P)}\left(\mid \mathbf{r}_{1}-\right.$ $\left.\mathbf{r}_{2} \mid, \omega\right)=\delta\left(\left|\mathbf{r}_{1}-\mathbf{r}_{2}\right|\right) \delta_{i j}$; i.e., they are $\delta$ correlated and follow the fluctuation-dissipation theorem [39].

On inserting Eq. (4) into (1) and taking the statistical homogeneity and isotropy of the source into account, one obtains the total force on the nanoparticle due to the random field inciding on it after emission. Only the force along the $z$ axis is different from zero, namely, the mechanical action of the source on the particle is rotationally symmetric as a consequence of its statistical isotropy. The conservative (gradient) part of the electric force $[28,33]\left\langle F_{i}^{e, \text { cons }}\right\rangle$, associated to this incident field $E_{j}^{\text {inc }},\left\langle F_{i}^{e, \text { cons }}\right\rangle=\varepsilon_{0} \operatorname{Re} \alpha_{e} \partial_{i}\left\langle E_{j}^{\text {inc, } *}(\mathbf{r}) E_{j}^{\text {inc }}(\mathbf{r})\right\rangle / 4$ is (cf. Appendix A)

$$
\begin{aligned}
\left\langle F_{z}^{e, \text { cons }}\right\rangle= & -\frac{k_{0}^{4} \pi}{8 \varepsilon_{0}} \operatorname{Re} \alpha_{e} \int^{(P)}(\omega) \int_{K=k_{0}}^{K=+\infty} \frac{\sqrt{K^{2}-k_{0}^{2}}}{\left|\gamma_{1}\right|^{2}} e^{-\frac{(K \sigma)^{2}}{2}} \\
& \times\left[\left|t_{12}^{s}\right|^{2}+\frac{\left|t_{12}^{p}\right|^{2}}{\left|n_{1}\right|^{2}\left|n_{2}\right|^{2} k_{0}^{4}}\left(\left|\gamma_{2}\right|^{2}+K^{2}\right)\left(\left|\gamma_{1}\right|^{2}+K^{2}\right)\right] \\
& \times e^{-2 z_{0} \operatorname{Im} \gamma_{2}} \frac{1}{\operatorname{Im} \gamma_{1}} e^{-\frac{1}{2} \sigma^{2} \operatorname{Re} \gamma_{1}^{2}} K d K
\end{aligned}
$$

where $\mathbf{K}=\left(K_{x}, K_{y}\right), \hat{\mathbf{s}}=\hat{\mathbf{K}} \times \hat{\mathbf{z}}, \hat{\mathbf{p}}_{i}^{ \pm}=-\left[\gamma_{i} \hat{\mathbf{K}} \mp K \hat{\mathbf{z}}\right] /\left(n_{i} k_{0}\right)$, and $\gamma_{i}=\sqrt{\varepsilon_{i} \mu_{i} k_{0}^{2}-K^{2}}(i=1,2) \cdot n_{1}\left(n_{2}\right)$ is the refractive index of the medium placed at $z<0(z>0)$ and the caret denotes a unit vector. It is worth stressing that the integration range in the above equation contribution is solely due to the evanescent modes [see also Eqs. (21) and (27) of Appendix A].

At this point it should be remarked that the main result of [5] is only an approximation to Eq. (5) for the electric force in the limit $K \rightarrow k_{0}$ for a Rayleigh particle considered in the quasistatic limit, i.e., that with $\alpha_{e}=a^{3}\left(\epsilon_{p}-1\right) /\left(\epsilon_{p}+2\right)$, which does not conserve energy on interaction with the random field [33]. By contrast, Eq. (5) accounts for all energy-conserving and retarded effects that are required for any general study, including those that otherwise could not be tackled when a larger nanoparticle, like a magnetodielectric one, is addressed.

On the other hand, the nonconservative part of the electric force $[28,33],\left\langle F_{i}^{e, \text { nc }}\right\rangle=\varepsilon_{0} \operatorname{Im} \alpha_{e} \operatorname{Im}\left\langle E_{j}^{\mathrm{inc}, *}(\mathbf{r}) \partial_{i} E_{j}^{\mathrm{inc}}(\mathbf{r})\right\rangle / 2$, is determined in a similar way [see Eq. (28) of Appendix A]:

$$
\begin{aligned}
\left\langle F_{z}^{e, \mathrm{nc}}\right\rangle= & \frac{k_{0}^{4} \pi}{8 \varepsilon_{0}} \operatorname{Im} \alpha_{e} \int^{(P)}(\omega) \int_{K=0}^{K=k_{0}} \frac{\sqrt{k_{0}^{2}-K^{2}}}{\left|\gamma_{1}\right|^{2}} e^{-\frac{(K \sigma)^{2}}{2}} \\
& \times\left[\left|t_{12}^{s}\right|^{2}+\frac{\left|t_{12}^{p}\right|^{2}}{\left|n_{1}\right|^{2}\left|n_{2}\right|^{2} k_{0}^{4}}\left(\left|\gamma_{1}\right|^{2}+K^{2}\right)\left(\left|\gamma_{2}\right|^{2}+K^{2}\right)\right] \\
& \times \frac{1}{\operatorname{Im} \gamma_{1}} e^{-\frac{1}{2} \sigma^{2} \operatorname{Re} \gamma_{1}^{2}} K d K
\end{aligned}
$$

where it is seen that only the homogeneous (propagating) plane-wave components contribute and yield a constant nonzero value for any $\mathbf{r}$.

The magnetic and the interference forces [cf. second and third terms of Eq. (1)] are calculated analogously.

\section{B. Forces from the secondary sources constituted by the induced fluctuating dipoles of the nanoparticle}

As previously stated, the last two terms in Eq. (2) are the fields emitted by the induced dipoles in the particle, and hence connect the constitutive properties of the source and particle through the Fresnel reflection coefficients $r^{s, p}$ at $z=0$ and the polarizabilities, being described in this case by $G_{i j}^{E p}$ and $G_{i j}^{H m}$. Thus the electric field can be calculated like in Eq. (4) using $G_{i j}^{E p}$ and $p_{i} \delta\left(\mathbf{r}^{\prime}-\mathbf{r}_{0}\right)$ instead of $G_{i j}^{E P}$ and $P_{j}\left(\mathbf{r}^{\prime}\right)$, i.e.,

$$
\begin{aligned}
E_{i}^{p}(\mathbf{r}) & =\mu_{0} \omega^{2} \int_{V} G_{i j}^{E p}\left(\mathbf{r}, \mathbf{r}^{\prime}, \omega\right) p_{j}\left(\mathbf{r}^{\prime}, \omega\right) \delta\left(\mathbf{r}^{\prime}-\mathbf{r}_{0}\right) d^{3} \mathbf{r}^{\prime} \\
& =\mu_{0} \omega^{2} G_{i j}^{E p}\left(\mathbf{r}, \mathbf{r}_{0}, \omega\right) p_{j}\left(\mathbf{r}_{0}, \omega\right),
\end{aligned}
$$

and analogously the electric field emitted by the induced magnetic dipole reads

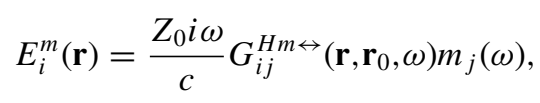

where the superscript $\leftrightarrow$ means that the electric field generated by the magnetic dipole has the same Green's function as the magnetic field radiated by the electric dipole with the interchange $r^{s} \leftrightarrow r^{p}$. Both $G_{i j}^{E(p, m)}$ and $G_{i j}^{H(p, m)}$ exponentially decay with the distance $z$ in the evanescent wave region 
$\left(K>k_{0}\right)$ and are oscillatory in the radiative one $\left(K \leqslant k_{0}\right)$. They are obtained as indicated in Appendix B.

Note that $r^{p}$ and $t^{p}$ support SPPs. The calculations are done considering that there is mutual incoherence between the nanoparticle electric and magnetic induced dipoles, i.e., $\left\langle p_{i}^{*} m_{j}\right\rangle=0$ [39]. In the following, $\left\langle F_{1}\right\rangle$ and $\left\langle F_{2}\right\rangle$ will denote the total forces [cf. Eq. (1)] due to the above-mentioned contributions of the primary fluctuating source in $z<0$ and of the secondary source constituted by the particle induced dipoles, respectively.

\section{EXAMPLE: SEMICONDUCTOR SPHERE IN THE NEAR INFRARED AND VISIBLE}

We illustrate the above with a generally magnetodielectric dipolar nanoparticle constituted by a semiconductor sphere; its anomalous scattering properties have recently received a great deal of attention, both theoretically and experimentally [20,27,40-42]. In particular, for each plane-wave component of the field incident on the particle, its scattered intensity in the backscattering direction is zero [first Kerker condition (K1)] when $\operatorname{Re} \alpha_{e}=\operatorname{Re} \alpha_{m}$. Also, for each of these incident planewave components, the forwardly scattered intensity becomes close to a nonzero minimum [second Kerker condition (K2)] when $\operatorname{Re} \alpha_{e}=-\operatorname{Re} \alpha_{m}$. There being no gains in the particle, in both cases: $\operatorname{Im} \alpha_{e}=\operatorname{Im} \alpha_{m}$ [40].

After performing all the integrations of the form of Eq. (4) for the primary source and for the induced electric and magnetic dipoles that constitute the above-mentioned secondary source, a long but straightforward task some of the details of which are shown in the Appendixes, one sees that $\left\langle E_{i}^{m *}\left(\mathbf{r}_{0}\right) E_{i}^{m}\left(\mathbf{r}_{0}\right)\right\rangle=\left\langle H_{i}^{p *}\left(\mathbf{r}_{0}\right) H_{i}^{p}\left(\mathbf{r}_{0}\right)\right\rangle=0$. This is relevant in connection with the Kerker conditions.

\section{A. Results for a silicon sphere. Forces from the primary source}

Let the sphere be made of Si with radius $a=230 \mathrm{~nm}$, with the incident light being in the NIR range of 1.2-2 $\mu \mathrm{m}$. At these wavelengths the total cross section of the nanoparticle is fully determined by the electric and magnetic Mie coefficients $a_{1}$ and $b_{1}$ [19], respectively. This justifies the use of Eq. (1) for the optical force.

In the configuration addressed here (cf. Fig. 1), the surface at $z=0$ is assumed to be metallic supporting surface plasmon polaritons (SPPs) for $p$ polarization, i.e., at $K=$ $k_{0} \sqrt{\varepsilon_{1} /\left(\varepsilon_{1}+1\right)}$ there is a pole in the correspondent Fresnel coefficient [38] (for $\varepsilon_{1}<1$ ). We consider an Au interface, hence supporting SPPs in the spectral range under consideration. It is worth remarking, however, that the presence of SPPs on $z=0$ is considered here because it enhances by one order the magnitude the strength scale of the different force components, but it does not produce any qualitative change in their relative behaviors [34]. Results are shown in the NIR; nonetheless, it is worth stressing that the scaling property of these high permittivity particles leads to identical results ranging from visible to microwave regions just by appropriately changing the size and permittivity of the particle (cf. Fig. 2 of [19] and also $[25,41,42])$.

Figure 2 shows the force terms, in a logarithmic scale, due to the fluctuating primary source and to the induced electric and magnetic random dipoles in the particle. This representation aims to clarify its extremely sharp changes of sign. Unless stated otherwise, in order to see the relative weight of each force component, all results of this paper are normalized to the power spectrum $\int^{(P)}(\omega)$ of the source. The first horizontal row shows the force $\left\langle F_{1}\right\rangle$ due to the field impinging the sphere from the primary statistical source at $z=0$. The second horizontal row represents the force $\left\langle F_{2}\right\rangle$ from the secondary
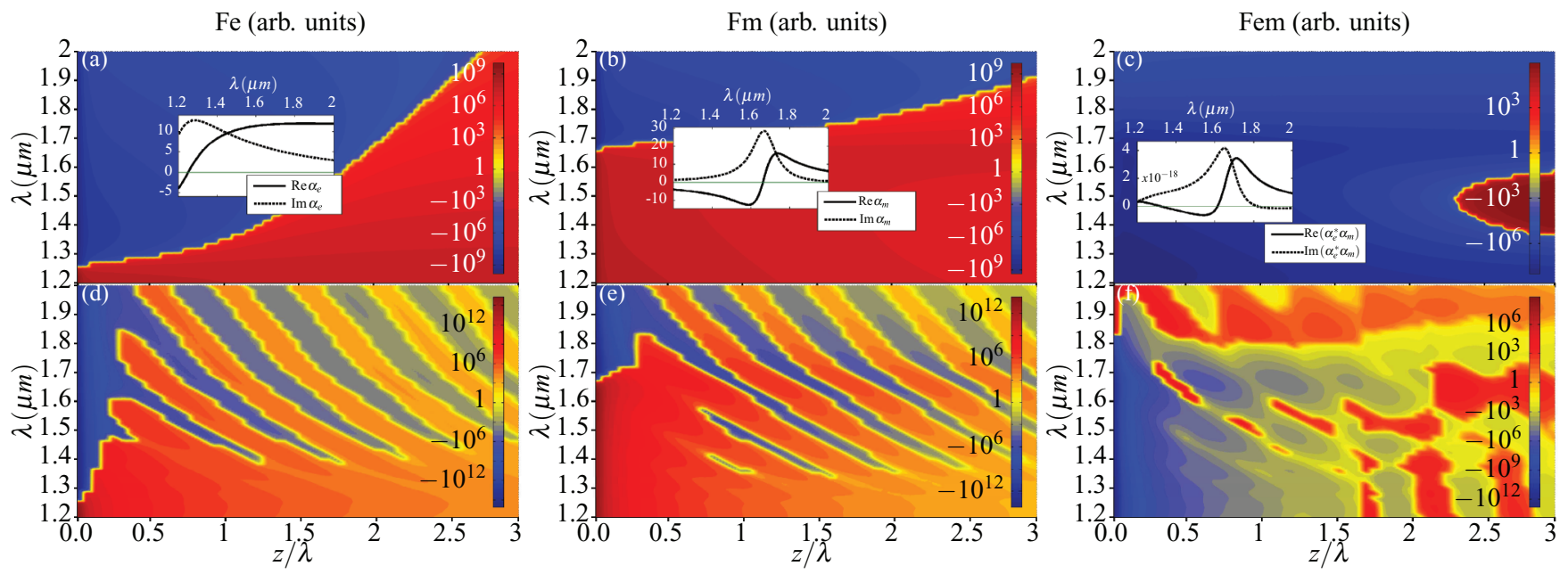

FIG. 2. (Color online) From left to right: Normalized electric $\left\langle F^{e}\right\rangle$, magnetic $\left\langle F^{m}\right\rangle$, and interference force $\left\langle F^{e m}\right\rangle$ from a $\delta$-correlated source. The first horizontal row shows the part of the force due to the field from the fluctuating primary source whose exit plane is at $z=0$ [first term in Eq. (2)]. The insets exhibit the polarizabilities normalized to $a^{3}$ vs $\lambda$ : electric, magnetic, and electric-magnetic product, respectively, which also depict the behavior of the corresponding force from each plane-wave component [33], and hence $\alpha_{e}, \alpha_{m}$, and $\alpha_{e}^{*} \alpha_{m}$ are factors in $\left\langle F^{e}\right\rangle$, $\left\langle F^{m}\right\rangle$, and $\left\langle F^{e m}\right\rangle$, respectively. The second horizontal row represents the force from the secondary source constituted by the particle induced dipoles [second and third terms in Eq. (2)]. In each figure, the warm and cold color regions, separated by a yellow line of minimum force strength, correspond to the zones where the force is positive and negative, respectively. The normalization of these forces is done on dividing their value by the power spectrum $\int^{(P)}(\omega)$ of the source. 
source constituted by the particle induced fluctuating electric and magnetic dipoles. The source coherence length $\sigma$ is first assumed to be zero. The inset depicts the polarizability contributions for the range of wavelengths under which it exhibits a resonant behavior; this helps one to understand the color plots.

In Figs. 2(a) and 2(b) we see a yellow line separating the gradient and scattering forces. For a statistically homogeneous source, the gradient force [proportional to $\left.\operatorname{Re} \alpha_{e}\left\langle E_{i}^{*} E_{i}\right\rangle\right]$ is governed uniquely by the evanescent modes and is negative for a particle with $\operatorname{Re} \alpha_{e}>0$, [cf. [34] and Eq. (5)]; hence it exponentially decays with the distance $z$ to the source. On the other hand, the scattering force (proportional to $\left.\operatorname{Im} \alpha_{e} \operatorname{Im}\left\langle E_{j}^{*} \partial_{i} E_{j}\right\rangle\right)$ is positive, i.e., pushing, and constant for any $\mathbf{r}_{0}$. As the wavelength grows, $\operatorname{Re} \alpha_{e}>\operatorname{Im} \alpha_{e}$ [see the inset in Fig. 2(a)]. This behavior and that corresponding to $\alpha_{m}$ shown in the inset of Fig. 2(b) results, as a consequence of the integrations in Eqs. (5) and (6), in an extraordinary phenomenon: the contribution of the evanescent waves to the gradient force, Eq. (5), is noticeable even at distances $z / \lambda>1$. Hence, pretty far away from the surface, the gradient force is dominant at shorter frequencies, a fact that applies to any source which behaves as quasimonochromatic [43] in this range of selected wavelengths. This is a remarkable feature of the forces introduced by the resonant nature of these particles that we put forward here.

Figure 2(c) represents the force component $\left\langle F_{1}^{e m}\right\rangle$ due to interference between the nanoparticle induced electric and magnetic dipoles. In the near field this is attractive for any wavelength, even at distances larger than $\lambda$, where the force is proportional to $-\operatorname{Re}\left(\alpha_{e}^{*} \alpha_{m}\right) S_{i}\left[\mathbf{S}=\operatorname{Re}\left(\mathbf{E}^{*} \times \mathbf{H}\right) / 2\right.$ denotes the Poynting vector, which is independent of the distance $z$ ]. Such a negative force is known as a pulling force, and its interest has increased in past years [44-46]. In this respect, Fig. 2(c) shows the relevant role of the magnetodielectric behavior of these particles, although in this latter specific case when the two other force components, electric and magnetic, are added this pulling effect becomes small. We state, however, that although not shown here for brevity, the sum of three components $\left\langle F^{e}\right\rangle$, $\left\langle F^{m}\right\rangle$, and $\left\langle F^{e m}\right\rangle$ yields a tractor force up to the distance $z \simeq 3 \lambda$ in the range $\lambda \simeq 1.7-2 \mu \mathrm{m}$. This is a different feature stemming from the magnetodielectric character of this particle.

Concerning the force $\left\langle F_{2}\right\rangle$ from the particle induced dipoles, we see in the second horizontal row of Fig. 2 that its amplitude exponentially decays with the distance $z$ to the primary source exit plane $z=0$, and its sign depends on that of the particle polarizability; nevertheless, the oscillatory behavior of the Green's function due to propagating plane wave components manifests in this force. We also observe that it is six orders of magnitude larger than its counterpart $\left\langle F_{1}\right\rangle$ from the primary fluctuating source, at least at subwavelength distances $z$. We shall later discuss this fact.

To get a deeper understanding, Fig. 3 represents $\left\langle F_{1}\right\rangle$ for some selected wavelengths and for two different source coherence lengths: $\sigma=0$ and $\sigma=\lambda / 4$. The huge sharp changes in the sign of the force at a given distance $z / \lambda$ are clearly seen. For a statistically homogeneous source, the electric and magnetic cross-spectral density tensors fulfill [47]

$$
\varepsilon_{0}\left\langle E_{i}^{*}\left(\mathbf{r}_{1}, \omega\right) E_{j}\left(\mathbf{r}_{2}, \omega\right)\right\rangle=\mu_{0}\left\langle H_{i}^{*}\left(\mathbf{r}_{1}, \omega\right) H_{j}\left(\mathbf{r}_{2}, \omega\right)\right\rangle,
$$
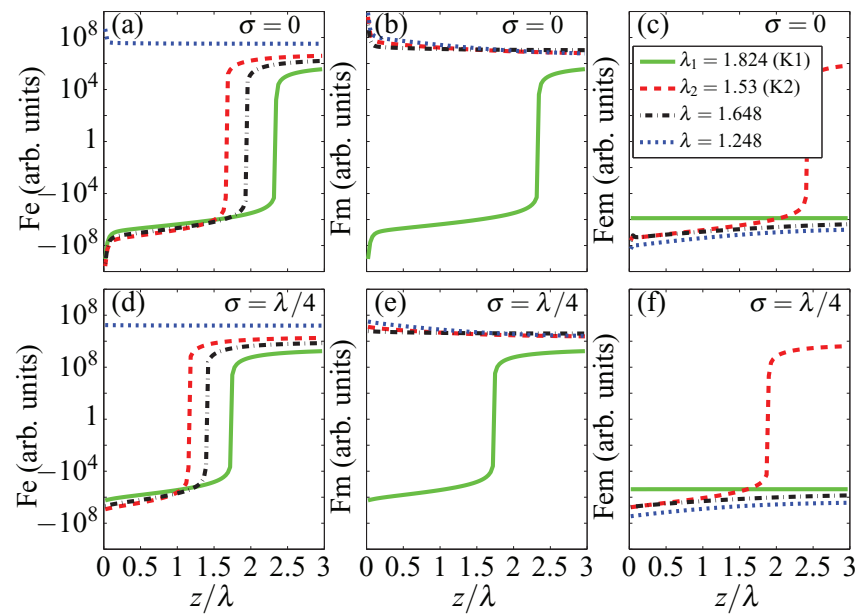

FIG. 3. (Color online) Normalized $\left\langle F_{1}^{e}\right\rangle,\left\langle F_{1}^{m}\right\rangle$, and $\left\langle F_{1}^{e m}\right\rangle$ vs the distance from the plane of the source (in wavelength units) for some values of the wavelength (in $\mu \mathrm{m}$ ). The two Kerker conditions occur at $\lambda_{1}(\mathrm{~K} 1)$ and $\lambda_{2}(\mathrm{~K} 2)$, respectively.

hence, in the near field one has $\left\langle F_{1}^{e}\right\rangle=\left\langle F_{1}^{m}\right\rangle$ and $\left\langle F_{1}^{e}\right\rangle=$ $-\left\langle F_{1}^{m}\right\rangle$ when the first and the second Kerker condition hold, respectively. However, in the far zone $\left\langle F_{1}^{e}\right\rangle=\left\langle F_{1}^{m}\right\rangle$ for any value of $\mathbf{r}_{0}$ at Kerker conditions. For the Si nanoparticle addressed, the Kerker conditions are fulfilled at $\lambda_{1} \simeq 1.825 \mu \mathrm{m}$ and $\lambda_{2} \simeq 1.53 \mu \mathrm{m}$ [cf. Figs. 2(a) and 2(b) and [40]].

Figure 4 depicts the range of wavelengths where the magnetic dipole predominates over the electric one. In the near field, where the electric (magnetic) force is solely due to the evanescent modes, the weight of such force is due to the response of the nanoparticle through its proportionality to the real part of the electric (magnetic) polarizability. Figure 4(a) exhibits a peak near $\lambda \simeq 1.25 \mu \mathrm{m}$, where the magnetic polarizability is more than one order of magnitude larger than its electric counterpart; thus the magnetic force will also surpass the electric one by one order of magnitude. We can also distinguish in this figure two zones close to $\lambda \simeq 1.6 \mu \mathrm{m}$ and $\lambda \simeq 1.76 \mu \mathrm{m}$ where $\left\langle F_{1}^{m}\right\rangle>\left\langle F_{1}^{e}\right\rangle$. On the other hand, the behavior of the force at larger distances will be ruled by the imaginary part of the polarizability. Figure 4(b) shows the zone where the magnetic dipole predominates, and thus the magnetic force is five times larger than the electric one. These effects, due to the magnetic response of the dielectric
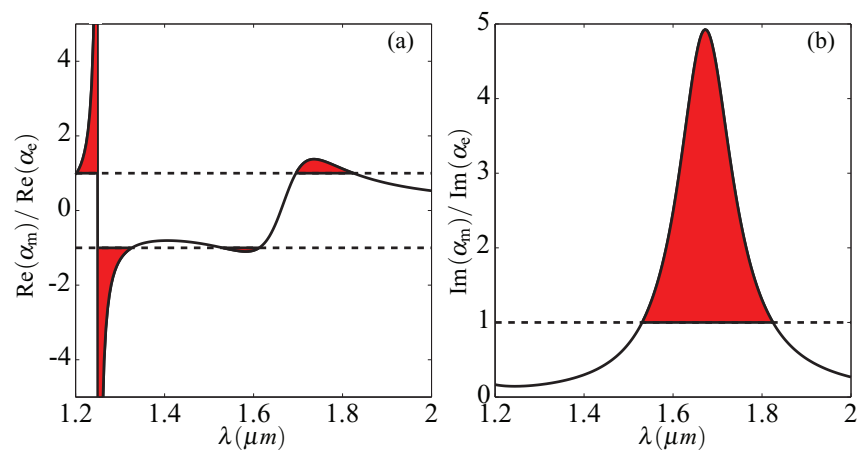

FIG. 4. (Color online) (a) $\operatorname{Re} \alpha_{m} / \operatorname{Re} \alpha_{e}$. (b) $\operatorname{Im} \alpha_{m} / \operatorname{Im} \alpha_{e}$. The colored areas denote the zones where the magnetic dipole predominates. 
particle to the light field, constitutes another main result of this paper.

\section{B. Influence of the coherence length and forces from the induced dipoles}

We now address the influence of the coherence length of the source. This establishes the differences between the mechanical action of our partially coherent optical sources and most previously studied that are $\delta$ correlated. In particular, at thermal wavelengths this would predict differences between Liftshitz, CP, and VdW forces from thermal sources and blackbodies, both in and out of thermodynamic equilibrium, and those generated from bodies whose currents are partially correlated.

In our study, the transversal part of the spectral degree of coherence (cf. Sec. II A) $\exp \left[-(K \sigma)^{2} / 2\right]$ governs the coherence tensors and the mean forces from the random field emitted by the primary source (see Appendix A) acting as a low-pass filter in $\mathbf{K}$ space [see Eqs. (5) and (6)], being maximum for $\sigma=0$, i.e., when the source is white noise. Because of this fact, the evanescent modes present two such filters: the first is due to their own nature, while the second stems from the spatial coherence of the source. The shape of Figs. 3(d)-3(f) is similar to that of Figs. 3(a)-3(c), shifted by a distance $\Delta z \simeq 0.5 \lambda$; therefore, for $\sigma>\lambda$ the force is solely due to the nonconservative (scattering) contribution and to the interference component $\left\langle F_{1}^{e m}\right\rangle$, which becomes constant and positive or negative depending on the wavelength. It is worth pointing out that the price paid on increasing the coherence length is expensive, because at the same time there is a reduction of the force strength by various orders of magnitude [cf., e.g., the forces shown in Figs. 3(a) and 3(d) at $z<\lambda$ ].

We now turn our study to analyze its influence on the force $\left\langle F_{2}\right\rangle$ generated by the secondary source, namely by the particle induced dipoles. Figure 5 represents $\left\langle F_{2}\right\rangle$ for the same wavelengths as in Fig. 3. The magnitude of this force $\left\langle F_{2}\right\rangle$ in the near-field $z<\lambda$ is much larger than that of $\left\langle F_{1}\right\rangle$ in Fig. 3; thus the effect of the mechanical action $\left\langle F_{2}\right\rangle$ of the field
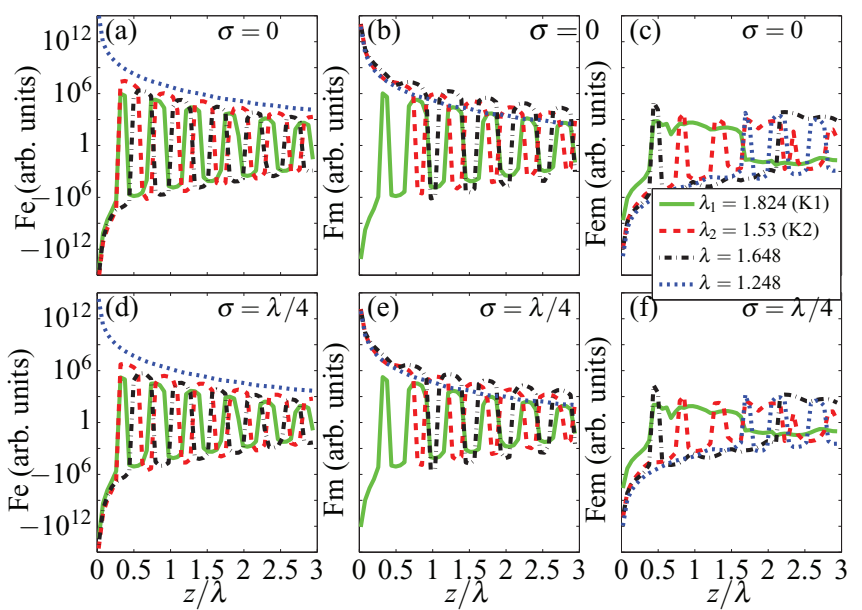

FIG. 5. (Color online) Normalized $\left\langle F_{2}^{e}\right\rangle,\left\langle F_{2}^{m}\right\rangle$, and $\left\langle F_{2}^{e m}\right\rangle$ versus distance $z$ (in units of wavelength) from the exit plane $z=0$ of the source for different values of the wavelength (in $\mu \mathrm{m}$ ). The two Kerker conditions are fulfilled at $\lambda_{1}(\mathrm{~K} 1)$ and $\lambda_{2}(\mathrm{~K} 2)$, respectively. emitted by the particle induced dipoles substantially dominates over that $\left\langle F_{1}\right\rangle$ of the field that is due solely to the primary fluctuating source. Nevertheless, as the distance $z$ grows, all the fastly oscillating components, electric, magnetic, and that of interference of this force $\left\langle F_{2}\right\rangle$ rapidly tend to zero, and hence the force $\left\langle F_{1}\right\rangle$ from the primary source is the one that dominates.

We remark that, as follows from the calculation of $E^{p, m}$ and $H^{p, m}$, the cross spectral density tensors of the electric and magnetic dipoles are not equal to each other; therefore, although at first sight it could seem that under Kerker conditions $\left\langle F_{2}^{e}\right\rangle$ and $\left\langle F_{2}^{m}\right\rangle$ would fulfill relationships similar to those of $\left\langle F_{1}^{e}\right\rangle$ and $\left\langle F_{1}^{m}\right\rangle$, in fact they do not. This is seen in Fig. 5.

The role of the coherence length in this case is exactly the same as in Fig. 2; the magnitude of the force decreases as $\sigma$ grows. Future work should find a minimum value of $\sigma$ for which this optical analogous to the $\mathrm{CP}$ force predominates over the contributions discussed here.

In order to provide an estimation of the actual magnitude of these forces, now instead of studying their relative values by using a normalization to $\int^{(P)}(\omega)$ as before, we evaluate them in terms of the optical power $I_{0}$ of the source. To this end, we consider a Gaussian quasimonochromatic spectrum [1] centered at frequency $\omega_{0}$ and spectral width $\sigma_{\omega}=0.01 \omega_{0}: \int^{(P)}(\omega)=$ $\left(\varepsilon_{0} / c k_{0}^{3}\right)\left(I_{0} / \sqrt{2 \pi} \sigma_{\omega}\right) \exp \left[-\left(\omega-\omega_{0}\right)^{2} / 2 \sigma_{\omega}^{2}\right]$, where $I_{0}$ is the optical power in $\mathrm{W} / \mathrm{m}^{2}$. Let this power be $I_{0}=1 \mathrm{~mW} / \mu \mathrm{m}^{2}$.

The resulting force exerted by the random field on the particle is determined by $\omega$ integration of all frequency components in the support of $\int^{(P)}(\omega)$, i.e., $F_{z}(\mathbf{r})=\int F_{z}(\mathbf{r}, \omega) d \omega$. [Compare Eqs. (5) and (6) for $F_{z}(\mathbf{r}, \omega)$. Notice that in the integrand we have now explicitly written the $\omega$ dependence of the force at each frequency $\omega]$.

First we calculate the forces $\left\langle F_{1}\right\rangle$ due to the primary source assuming it to be $\delta$ correlated. As seen in Fig. 3, the maximum magnitude of the force (independently of whether its origin is from the electric, magnetic, or electric-magnetic interference dipoles) occurs at subwavelength distances $z$ where the evanescent modes are more relevant. For instance, for $\lambda_{0}=1.6 \mu \mathrm{m}\left(\lambda_{0}=2 \pi c / \omega_{0}\right)$, the particle being at $z<$ $\lambda_{0} / 2$, the magnitude (in absolute value) of the electric force $\left\langle F_{1}^{e}\right\rangle$ is in the interval: $\left[1 \times 10^{-14}, 1 \times 10^{-13} \mathrm{~N}\right]$; and as the distance $z$ of the particle to the source increases, this electric force tends to a constant value (governed by the propagating modes) in this interval. Obviously, the sign of the force will be frequency dependent through the polarizability of the particle.

Analogously, and as a consequence of Eq. (9), the behavior of the magnitude of the magnetic force $\left\langle F_{1}^{m}\right\rangle$ is similar to that of $\left\langle F_{1}^{e}\right\rangle$. At the same distance $z$, the interference force $\left\langle F_{1}^{e m}\right\rangle$ is slightly different to these former. Indeed, as one sees on comparing Figs. 3(b) and 3(c), $\left\langle F_{1}^{e m}\right\rangle$ is two orders of magnitude smaller than either the electric or magnetic forces.

Subsequently, this calculation is performed for the forces $\left\langle F_{2}\right\rangle$ induced by the secondary source (i.e., from the induced dipoles in the particle). The same parameters as before are assumed for the primary source. As pointed out above in this section, at subwavelength distances $z$, these forces are much larger than those $\left\langle F_{1}\right\rangle$ from the primary source. At a distance $z \simeq \lambda_{0} / 10$, the electric force $\left\langle F_{2}^{e}\right\rangle$ is of the order of $10^{-12} \mathrm{~N}$, a value which is certainly larger than the aforementioned one 
for $\left\langle F_{1}^{e}\right\rangle$. On the other hand, at a distance $z \simeq \lambda_{0} / 4,\left\langle F_{1}^{e}\right\rangle$ starts to compete with $\left\langle F_{2}^{e}\right\rangle$, the latter becoming negligible at distances comparable to the wavelength $\lambda_{0}$ of the emitted field. Similar effects are found for the other two components $\left\langle F_{2}^{m}\right\rangle$ and $\left\langle F_{2}^{e m}\right\rangle$.

All these forces diminish when the coherence length $\sigma$ of the primary source increases. For instance, when $\sigma=\lambda / 4$, they all become about three to four orders of magnitude smaller than those previously obtained for a $\delta$-correlated source.

\section{ASYMPTOTIC LAWS IN THE NEAR FIELD}

\section{A. Fields from the primary source}

In this section we discuss the asymptotic behavior of the forces due to the field emerged from the primary source when we are at extreme near-field distances, $(z \ll \lambda)$, and for different correlation lengths $\sigma$. This analysis in the optical range makes a contact with previous studies of $\mathrm{CP}, \mathrm{VdW}$, and out of equilibrium forces corresponding to broad spectra like those from thermal or blackbody sources, and shows their analogies as well as the limitations of applying to particles previous studies on atoms.

In this connection, we stress the different sign of these forces in the region $z \ll \lambda$, either attractive or repulsive, shown in Figs. 3 and 5, depending on the sign of the particle polarizability according to the emission wavelength.

Multiple studies have led to a rather large landscape of $\mathrm{CP}$ forces depending on both the nature of the fluctuations and the electromagnetic properties of the sources [48]. We address the interaction from the polarization currents of the primary source (the magnetic currents are negligible), and the nanoparticle, generally considered as magnetodielectric.

At distances $z \ll \lambda$ the main contribution to the integral Eq. (5) comes from large values of $K$, i.e., $K \gg k_{0}$. Notice that, in this case, the total electric force is solely due to the conservative force $F_{z}^{e \text {,cons }}$ and consequently only the evanescent modes contribute.

We shall now consider two regimes in the quasistatic approximation $K \gg k_{0}$ [49]: the first is when $\sigma \rightarrow 0$. This is the most studied one because it can be extrapolated to a thermal source whose fluctuations come from Rytov's theory [39]. Thus, in this case, Eq. (5) leads to

$$
F_{z}^{e, \text { cons }} \simeq-\frac{3 \pi}{4 \varepsilon_{0} z^{4}} \operatorname{Re} \alpha_{e} \frac{\int^{(P)}(\omega)}{\left|\varepsilon_{1}+1\right|^{2}} \quad(z \ll \lambda, \sigma \rightarrow 0) .
$$

As we can see, the force decays as $1 / z^{4}$ and its sign depends on the sign of the polarizability of the nanoparticle, which can be positive (negative) leading to a negative (positive) force, respectively. This is analogous to the well-known quasistatic dependence of the $\mathrm{VdW}$ force when $\int^{(P)}(\omega)$ is the wide Planck's spectrum.

The second regime will be when $z \ll \lambda$ and $\sigma>z$; in this case the electric force is given by

$$
F_{z}^{e, \text { cons }} \simeq-\frac{4 \pi}{\varepsilon_{0} \sigma^{4}} \operatorname{Re} \alpha_{e} \frac{\int^{(P)}(\omega)}{\left|\varepsilon_{1}+1\right|^{2}} \quad(z \ll \lambda, \sigma>z) .
$$

Now the source with a nonzero coherence length $\sigma$ gives rise to a force which does not depend on the distance $z$, i.e., for an arbitrary value of $\sigma$, the force is constant with distance and the role of $z$ that appeared in Eq. (10) is now played by $\sigma$. Notice also that when $\sigma \gg \lambda>z$ the resulting force is negligible.

At this point we wish to point out that the conservative forces obtained in Eqs. (10) and (11) are compatible with recent results on spatial correlations of speckle patterns at extremely close distances from disordered media interfaces (cf. Sec. IV of [49]).

The conservative force $F_{z}^{m \text {,cons }}$ on the magnetic dipole, induced in the particle by the field emitted from the fluctuating primary source, is similarly calculated. As stated before, these electric and magnetic forces hold:

$$
F_{z}^{m, \text { cons }}=\frac{\operatorname{Re} \alpha_{m}}{\operatorname{Re} \alpha_{e}} F_{z}^{e, \text { cons }} .
$$

Next we calculate the term $F_{i}^{e m}$ of the force due to the interference between the induced electric and magnetic dipoles in the particle. At these distances, this force is solely due the evanescent modes of the angular spectrum as in the case of electric and magnetic force. We have

$$
\begin{gathered}
F_{i}^{e m}(\mathbf{r}) \simeq-\frac{k_{0}^{5}}{24 \varepsilon_{0} z^{2}} \operatorname{Im}\left(\alpha_{e}^{*} \alpha_{m}\right) \frac{\int^{(P)}(\omega)}{\left|\varepsilon_{1}+1\right|^{2}} \quad(z \ll \lambda, \sigma \rightarrow 0), \\
F_{i}^{e m}(\mathbf{r}) \simeq-\frac{k_{0}^{5} \pi}{6 \varepsilon_{0} \sigma^{2}} \operatorname{Im}\left(\alpha_{e}^{*} \alpha_{m}\right) \frac{\int^{(P)}(\omega)}{\left|\varepsilon_{1}+1\right|^{2}} \quad(z \ll \lambda, \sigma>z) .
\end{gathered}
$$

In contrast with the corresponding Eqs. (10) and (11), the interference force decays with the distance as $1 / z^{2}$, or with the coherence length as $1 / \sigma^{2}$.

We now turn to study the opposite asymptotic case, i.e., that of larger distances $z>\lambda$; this excludes any quasistatic approximation. At such large $z / \lambda$, one may expect almost no contribution of evanescent plane-wave components and thus, if as in [5] one approximated $K \rightarrow k_{0}$ in the integration of Eq. (5), one would obtain, in analogy with the calculation leading to Eqs. (10) and (11), an asymptotic power law for the conservative force, either electric [5], magnetic, or electricmagnetic interference, which, depending on the value of $\sigma$, would decay as $\propto-1 / z^{3}$ or $\propto-1 / \sigma^{3}$.

However, in the range $z>\lambda$, the nonconservative component of the force Eq. (6) is no longer negligible; hence, contrary to what might initially be thought, the conservative component is not enough to describe the total force on the particle at such distances. This completely changes the variation with $z$ of the resulting force which, as a consequence of adding the conservative and nonconservative components, is no longer of the form $\propto-1 / z^{3}$ or $\propto-1 / \sigma^{3}$. Notice that the existence of the nonconservative force in this regime of $z / \lambda$ is a consequence of the fact that, as mentioned before, the particle polarizabilities cannot be described by their quasistatic expressions.

To clarify this point, Fig. 6 shows the different components of the force in two axes. At these distances $z$, the exact expression Eq. (5) of $F_{z}^{e, \text { cons }}$ leads to the asymptotic power law $\propto-1 / z^{3}$ [5]. Nevertheless, on taking also into account $F_{z}^{e, n c}$ [cf. Eq. (6)], the total electric force $F_{z}^{e}=F_{z}^{e, \text { cons }}+F_{z}^{e, n c}$ no longer follows the power law $-1 / z^{3}$, but practically varies proportionally as $\simeq 1.33 z$ and becomes repulsive. This result points out the inadequacy of extrapolating previous quasistatic 


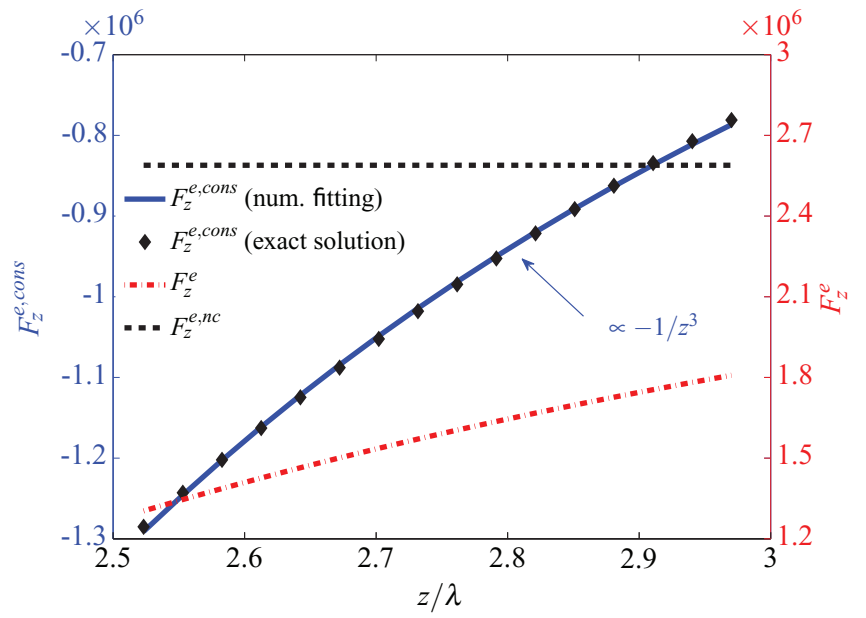

FIG. 6. (Color online) Components of the electric force at $\lambda=$ $1.63 \mu \mathrm{m}$. The diamonds and the blue-solid line (left axis) represent the exact solution and the numerical fitting $-1 / z^{3}$, respectively, for the conservative component [cf. Eq. (5)]. The dashed lines (right axis) depict the nonconservative force [cf. Eq. (6), black dashed line] and the total force $F_{z}^{e}=F_{z}^{e \text {,cons }}+F_{z}^{e, \text { nc }}$, (red dash-dot line). Notice how this latter sum makes the asymptotic behavior $-1 / z^{3}$ of $F_{z}^{e, \text { cons }}$ disappear, becoming almost linear with $z$ in the resulting total force $F_{z}^{e}$.

studies made for atoms to the analysis of the mechanical action on particles from both optical and thermal radiation $[4,5]$.

\section{B. Fields from the nanoparticle induced dipoles}

We now proceed with the interaction from the fluctuating induced dipoles. The electric force will now be proportional to $\operatorname{Re}\left\langle\alpha_{e} E_{j}^{p}(\mathbf{r}) \partial_{i} E_{j}^{p *}(\mathbf{r})\right\rangle$ (with an analogous expression for the magnetic and electric-magnetic interference forces). Now,

$$
\begin{aligned}
\left\langle E_{j}^{p *} \partial_{i} E_{j}^{p}\right\rangle & =\mu_{0}^{2} \omega^{4}\left\langle G_{j k}^{p *}\left(\mathbf{r}, \mathbf{r}^{\prime}\right) \partial_{i} G_{j l}^{p}\left(\mathbf{r}, \mathbf{r}^{\prime}\right)\right\rangle\left\langle p_{k}^{*}\left(\mathbf{r}^{\prime}\right) p_{l}\left(\mathbf{r}^{\prime}\right)\right\rangle \\
& =k_{0}^{4}\left|\alpha_{e}\right|^{2}\left\langle G_{j k}^{p *}\left(\mathbf{r}, \mathbf{r}^{\prime}\right) \partial_{i} G_{j l}^{p}\left(\mathbf{r}, \mathbf{r}^{\prime}\right)\right| I_{k l}\left(\mathbf{r}^{\prime}, \mathbf{r}_{1}\right),
\end{aligned}
$$

where $I_{k l}\left(\mathbf{r}^{\prime}, \mathbf{r}_{1}\right)=\left\langle E_{k}^{\text {inc } *}\left(\mathbf{r}^{\prime}, \mathbf{r}_{1}\right) E_{l}^{\text {inc }}\left(\mathbf{r}^{\prime}, \mathbf{r}_{1}\right)\right\rangle, \mathbf{r}, \mathbf{r}^{\prime}$ denote points of the half-space $z>0$, where the dipole particle is placed, while $\mathbf{r}_{1}$ stands for a point of $z<0$, (cf. Fig. 1). Note that Eq. (15) differs from that employed for calculating the vacuum fluctuations and the CP force because in Eq. (15) the particle induced dipoles are expressed in terms of the incident field and not by the usual fluctuation-dissipation theorem [39]. Now, to calculate the asymptotic behavior of the force, we have to approximate two Green's functions, one for the field which is transmitted from the primary source and another for the field which is reflected at $z=0$. As for the Green's function $G^{E P}$, we make the same approximations as in the previous section. On the other hand, for the Green's function $G^{E p}$, one can approximate the reflection Fresnel coefficients by [50]

$$
r_{s} \simeq \frac{k_{0}^{2}\left(\varepsilon_{1}-1\right)}{4 K^{2}}, \quad r_{p} \simeq \frac{\varepsilon_{1}-1}{\varepsilon_{1}+1} .
$$

Taking these considerations into account, we address the first asymptotic laws for this type of configuration. Thus, for the electric force, we get in the quasistatic approximation

$$
\begin{array}{r}
F_{z}^{e, \text { cons }}(\mathbf{r}) \simeq-\frac{\left|\alpha_{e}\right|^{2}}{128 \varepsilon_{0} z^{10}} \operatorname{Re} \alpha_{e} \int^{(P)}(\omega) \frac{\left|\varepsilon_{1}-1\right|^{2}}{\left|\varepsilon_{1}+1\right|^{4}} \\
(z \ll \lambda, \sigma \rightarrow 0), \\
F_{z}^{e, \text { cons }}(\mathbf{r}) \simeq-\frac{\sqrt{2 \pi}\left|\alpha_{e}\right|^{2}}{64 \varepsilon_{0} z^{7} \sigma^{3}} \operatorname{Re} \alpha_{e} \int^{(P)}(\omega) \frac{\left|\varepsilon_{1}-1\right|^{2}}{\left|\varepsilon_{1}+1\right|^{4}} \\
(z \ll \lambda, \sigma>z) .
\end{array}
$$

Thus showing dependence on the distance either as $1 / z^{10}$ or $1 / z^{7}$. Therefore, there is a difference of three orders of magnitude between the incoherent $(\sigma \rightarrow 0)$ and the partially coherent $\sigma>0$ limits. This difference is given by $I_{k l}$ which contains the coherence length $\sigma$ of the source. At these small distances, the nonconservative components of the forces are zero.

In a similar way, we derive the expression for the force from the particle induced magnetic dipole. In this case, we get

$$
\begin{gathered}
F_{z}^{m, \text { cons }}(\mathbf{r}) \simeq-\frac{k_{0}^{4}\left|\alpha_{m}\right|^{2}}{128 \varepsilon_{0} z^{6}} \operatorname{Re} \alpha_{m} \int^{(P)}(\omega) \frac{\left|\varepsilon_{1}-1\right|^{4}}{\left|\varepsilon_{1}+1\right|^{4}} \\
(z \ll \lambda, \sigma \rightarrow 0), \\
F_{z}^{m, \text { cons }}(\mathbf{r}) \simeq-\frac{\sqrt{2 \pi} k_{0}^{4}\left|\alpha_{m}\right|^{2}}{256 \varepsilon_{0} z^{3} \sigma^{3}} \operatorname{Re} \alpha_{m} \int^{(P)}(\omega) \frac{\left|\varepsilon_{1}-1\right|^{4}}{\left|\varepsilon_{1}+1\right|^{4}} \\
(z \ll \lambda, \sigma>z) .
\end{gathered}
$$

Equation (19) does not show the same decay with $z$ as Eq. (17) exhibits for the electric force; this shows that the induced magnetic dipole does not interact with the source plane at $z=0$ in the half-space $z>0$ in the same way as the particle induced electric dipole.

The expressions for the electric-magnetic interference force are a bit more complicated to analytically derive; however, making a numerical fitting, we see that it decays as $-1 / z^{8}$ or $-1 /\left(z^{5} \sigma^{3}\right)$ in the incoherent and partially coherent cases, respectively.

The asymptotic expression for these secondary source force components is not meaningful at larger distances. As remarked in Sec. III A, the forces from the particle induced dipole fields decay much faster than those from the primary source fields; thus the nonconservative component will predominate, being constant with distance $z$ as shown in Sec. III A. It should also be noticed that in order to recover the retarded typical power law $1 / z^{5}$ associated to CP forces from vacuum fluctuations [30], no primary source fluctuating fields should be present. As mentioned in the Introduction, $\hbar \omega / k T \gg 1$ at the frequencies considered, and hence if the source obeyed a Planck law the resulting spectral distribution would be the optical analogous to that of the vacuum fluctuations with energy $\hbar \omega / 2$ [8]; therefore, a short of switch-off of the emitted field would be necessary to detect such a vacuum fluctuation optical analogous force.

\section{CONCLUSIONS}

We have predicted some effects and asymptotic laws as regards radiation forces on a resonant dipolar nanoparticle due to NIR random light fields emitted by a partially coherent, statistically homogeneous random source. The particle has been 
considered to be a dielectric sphere of a kind that has recently received much attention due to its general magnetodielectric response to the illuminating field. The same applies in the visible or microwave regions just by appropriately changing the size and permittivity of the particle. For broadband sources, this range of frequencies makes these forces optical analogous to Casimir Polder (CP), Van der Waals (VdW), and to those out from thermodynamic equilibrium.

It has been shown that by manipulating the optical source, and in particular when it is quasimonochromatic, either pushing or attractive forces appear, even far from its emission plane. This creates new possibilities for nanoparticle ensembling and manipulation. Also, the magnetodielectric behavior of the sphere and its resonant response brings more force components into play than just those from the electric induced dipole; this creates a rich landscape of optical interactions as the central frequency of the emission spectrum varies. Hence, beyond $\mathrm{CP}$ and $\mathrm{VdW}$ effects, these induced dipole optical forces may be made negligible versus those from the primary source on monitoring the power of the latter. For example, far away from the source interface, the gradient force may become dominant for any quasimonochromatic source. Similarly, the magnetic force may dominate over that exerted by the usual nanoparticle induced electric dipole field. Even with small radiating powers, the range of these forces is of the order of pico-Newtons. Hence they are detectable and are larger than most thermal fluctuation forces.

Quasimonochromatic primary sources are the best candidates to observing the above-mentioned rich variety of wavelength-dependent forces. Also electric, magnetic, and interference force components have been separately shown in order to better analyze their respective nature and contribution to the total force. In this respect, we have emphasized that a frequency superposition to determine these forces, as usually done with thermal interactions, does not change their spatial dependence; however, it washes out their respective wavelength behavior.

Conversely, we have shown configurations where one may choose the frequency, or the spectral position and bandwidth of illumination, to tailor a predominance of either the induced dipole fluctuations (the optical analogous and generalizations of the $\mathrm{CP}$ or $\mathrm{VdW}$ forces), or those from the primary fluctuating source emitted field, in analogy with those thermal forces out of equilibrium.

Additionally, this study manifests the shortcomings of extending to previous particle studies on atoms near surfaces. This is seen in connection with the asymptotic forms of these radiation forces. These problems stem from the use of the quasistatic approximation, widely employed for atoms, to study the case of these nanoparticles. In this way, asymptotic power laws in the extreme proximity to the source are obtained for the conservative force, either electric, magnetic, or of electric-magnetic interference, which are of the form $-1 / z^{3}$ or $1 / \sigma^{3}$, according to whether the source coherence length $\sigma$ is null or not. In addition, at distances larger than the wavelength the quasistatic approximation fails and, contrary to the case of atoms near a surface, the nonconservative component of the force cannot be neglected and the attraction exerted by the source on the nanosphere no longer follows an inverse third power law, but linearly varies with the distance $z$.
On the other hand, a dependence on the distance to the source is found for the fields from the nanoparticle induced dipoles. This is shown to be $-1 / z^{10}$ or $-1 / z^{7}$, depending on whether the source coherence length $\sigma$ is zero or not.

Further experiments should be stimulated by these new effects, including consequences of other resonances like those of Fano.

\section{ACKNOWLEDGMENTS}

Research financed by Ministerio de Economia y Competitividad (MINECO) through the Consolider NanoLight CSD2007-00046 and FIS2012-36113-C03-03 research grants. J.M.A. acknowledges a scholarship from MINECO. C.W.Q. acknowledges the financial support by National University of Singapore (Grant No. R-263-000-678-133).

\section{APPENDIX A: GREEN'S FUNCTION $G_{i j}^{E P}$ AND SELF-CORRELATION FUNCTION $\left\langle E_{j}^{\text {inc } *}(r) E_{j}^{\text {inc }}(r)\right\rangle$}

The Green's function which relates the properties of the source placed at $z<0$ and the free propagation in $z>0$ is expressed on using Weyl's expansion in terms of an angular spectrum of plane waves [1,32,37,38]:

$$
G_{i j}^{E P}\left(\mathbf{r}, \mathbf{r}^{\prime}, \omega\right)=\frac{i}{2} \int_{-\infty}^{\infty} \frac{d^{2} \mathbf{K}}{(2 \pi)^{2}} G_{i j}^{E P}(\mathbf{K}) e^{i \mathbf{K}\left(\mathbf{R}-\mathbf{R}^{\prime}\right)} e^{i \gamma_{2} z-i \gamma_{1} z^{\prime}} .
$$

$G_{i j}^{E P}(\mathbf{K})=\frac{1}{\gamma_{1}}\left(\hat{s}_{i} t_{12}^{s} \hat{s}_{j}+\hat{p}_{1 i}^{+} t_{12}^{p} \hat{p}_{2 j}^{+}\right) . \quad \gamma_{i}=\sqrt{\varepsilon_{i} \mu_{i} k_{0}^{2}-K^{2}}$ if $\varepsilon_{i} \mu_{i} k_{0}^{2} \geqslant K^{2}$ (homogeneous waves), and $\gamma_{i}=i \sqrt{K^{2}-\varepsilon_{i} \mu_{i} k_{0}^{2}}$ if $\varepsilon_{i} \mu_{i} k_{0}^{2}<K^{2}$ (evanescent waves) $(i=1,2)$. The vectors $\hat{\mathbf{s}}$ and $\hat{\mathbf{p}}$ are defined in the main text. The superindices $s$ and $p$ denote the Fresnel transmission coefficient $t_{12}$ from region 1 to region 2 through $z=0$ under $s$ and $p$ polarization, respectively. Thus the function $\left\langle E_{j}^{\mathrm{inc} *}(\mathbf{r}) E_{j}^{\mathrm{inc}}(\mathbf{r})\right\rangle$ will be

$$
\begin{aligned}
\left\langle E_{j}^{\mathrm{inc} *}(\mathbf{r}) E_{j}^{\mathrm{inc}}(\mathbf{r})\right\rangle= & \mu_{0}^{2} \omega^{4} \int_{V_{1}, V_{2}} G_{j k}^{E P *}\left(\mathbf{r}, \mathbf{r}_{1}^{\prime}, \omega\right) G_{j l}^{E P}\left(\mathbf{r}, \mathbf{r}_{2}^{\prime}, \omega\right) \\
& \times W_{k l}^{(P)}\left(\mathbf{r}_{1}^{\prime}, \mathbf{r}_{2}^{\prime}, \omega\right) d^{3} \mathbf{r}_{1}^{\prime} d^{3} \mathbf{r}_{2}^{\prime} .
\end{aligned}
$$

Substituting Eq. (22) into Eq. (21) we have $\left\langle E_{j}^{\text {inc* }}(\mathbf{r}) E_{j}^{\text {inc }}(\mathbf{r})\right\rangle$ explicitly expressed in terms of the angular spectrum:

$$
\begin{aligned}
& \left\langle E_{j}^{\mathrm{inc} *}(\mathbf{r}) E_{j}^{\mathrm{inc}}(\mathbf{r})\right\rangle \\
& =\frac{\mu_{0}^{2} \omega^{4}}{4} \int_{V_{1}, V_{2}} \int_{-\infty}^{\infty} \frac{d^{2} \mathbf{K}_{1}}{(2 \pi)^{2}} \frac{d^{2} \mathbf{K}_{2}}{(2 \pi)^{2}} G_{j k}^{E P *}\left(\mathbf{K}_{1}\right) G_{j l}^{E P}\left(\mathbf{K}_{2}\right) \\
& \quad \times e^{-i \mathbf{K}_{1}\left(\mathbf{R}-\mathbf{R}_{1}^{\prime}\right)} e^{i \mathbf{K}_{2}\left(\mathbf{R}-\mathbf{R}_{2}^{\prime}\right)} e^{-\left(i \gamma_{2,1}^{*} z-i \gamma_{1,1}^{*} z_{1}^{\prime}\right)} \\
& \quad \times e^{i \gamma_{2,2} z-i \gamma_{1,2} z_{2}^{\prime}} W_{k l}^{(P)}\left(\mathbf{r}_{1}^{\prime}, \mathbf{r}_{2}^{\prime}, \omega\right) d^{3} \mathbf{r}_{1}^{\prime} d^{3} \mathbf{r}_{2}^{\prime}
\end{aligned}
$$

where $\gamma_{i, j}^{2}=\varepsilon_{i} \mu_{i} k_{0}^{2}-K_{j}^{2}$ and $(i, j)=1,2$.

Since, as seen in the main text, the cross-spectral density tensor is $W_{i j}^{(P)}\left(\mathbf{r}_{1}, \mathbf{r}_{2}, \omega\right)=\int^{(P)}(\omega) \exp \left[-\left(\mid \mathbf{r}_{1}-\right.\right.$ $\left.\left.\mathbf{r}_{2} \mid\right)^{2} / 2 \sigma^{2}\right] \delta_{i j} /(2 \pi)^{3 / 2} \sigma^{3}$, on writing the position vector, $\mathbf{r}_{n}=\left(\mathbf{R}_{n}, z_{n}\right), \mathbf{R}_{n}=\left(x_{n}, y_{n}\right)$ with $n=1,2$, we can express the following: $W_{i j}^{(P)}\left(\mathbf{r}_{1}, \mathbf{r}_{2}, \omega\right)=F\left(\left|\mathbf{R}_{1}-\mathbf{R}_{2}\right|, \omega\right) \exp \left[-\left(z_{1}-\right.\right.$ $\left.\left.z_{2}\right)^{2} / 2 \sigma^{2}\right]$, where we have written $F\left(\left|\mathbf{R}_{1}-\mathbf{R}_{2}\right|, \omega\right)=$ $\int^{(P)}(\omega) \exp \left[-\left(\left|\mathbf{R}_{1}-\mathbf{R}_{2}\right|\right)^{2} / 2 \sigma^{2}\right] \delta_{i j} /(2 \pi)^{3 / 2} \sigma^{3}$. 
We now make the change of variables: $\mathbf{R}_{1}^{\prime}=\mathbf{R}^{\prime \prime}+\mathbf{R}^{\prime} / 2$ and $\mathbf{R}_{2}^{\prime}=\mathbf{R}^{\prime \prime}-\mathbf{R}^{\prime} / 2$. Performing the $\mathbf{R}^{\prime \prime}$ integration which gives a two-dimensional $\delta$ function $\delta^{(2)}\left[\mathbf{K}_{1}-\mathbf{K}_{2}\right]$ and the $\mathbf{R}^{\prime}$ integration, Eq. (A3) leads to

$$
\begin{aligned}
& \left\langle E_{j}^{*}(\mathbf{r}) E_{j}(\mathbf{r})\right\rangle \\
& =\frac{\mu_{0}^{2} \omega^{4}}{4} \int_{-\infty}^{0} d z_{2}^{\prime} d z_{1}^{\prime} \int_{-\infty}^{\infty} d^{2} \mathbf{K} \frac{1}{\left|\gamma_{1}\right|^{2}} \tilde{F}(\mathbf{K}, \omega) \\
& \quad \times\left|G_{j k}^{E P *}(\mathbf{K})\right|^{2} e^{-2 z \operatorname{Im} \gamma_{2}} e^{i\left(\gamma_{1}^{*} z_{1}^{\prime}-\gamma_{1} z_{2}^{\prime}\right)} e^{-\left(z_{1}^{\prime}-z_{2}^{\prime}\right)^{2} / 2 \sigma^{2}} .
\end{aligned}
$$

$\tilde{F}(\mathbf{K}, \omega)$ is the two-dimensional Fourier transform of $F\left(\mid \mathbf{R}_{1}-\right.$ $\left.\mathbf{R}_{2} \mid, \omega\right)$. With $z_{1}^{\prime}=z^{\prime \prime}+z^{\prime} / 2, z_{2}^{\prime}=z^{\prime \prime}-z^{\prime} / 2$ and using the expression for $\tilde{F}(\mathbf{K}, \omega)$, the last equation becomes

$$
\begin{aligned}
\left\langle E_{j}^{*}(\mathbf{r}) E_{j}(\mathbf{r})\right\rangle= & \frac{\mu_{0}^{2} \omega^{4}}{8} \int^{(P)}(\omega) \int_{-\infty}^{\infty} \frac{1}{\left|\gamma_{1}\right|^{2}} e^{-\frac{(K \sigma)^{2}}{2}}\left|G_{j k}^{E P}(\mathbf{K})\right|^{2} \\
& \times e^{-2 z \operatorname{Im} \gamma_{2}} \frac{1}{\operatorname{Im} \gamma_{1}} e^{-\frac{1}{2} \sigma^{2} \operatorname{Re} \gamma_{1}^{2}} d^{2} \mathbf{K} .
\end{aligned}
$$

Next we use the following expression [37]:

$$
\left|G_{j k}^{E P}(\mathbf{K})\right|^{2}=\left|t_{12}^{s}\right|^{2}|\hat{\mathbf{s}}|^{2}|\hat{\mathbf{s}}|^{2}+\left|t_{12}^{p}\right|^{2}\left|\hat{\mathbf{p}}_{1}^{+}\right|^{2}\left|\hat{\mathbf{p}}_{2}^{+}\right|^{2},
$$

which taking into account $\hat{\mathbf{s}}$ and $\hat{\mathbf{p}}^{ \pm}$, defined after Eq. (5), becomes

$$
\left|G_{j k}^{E *}(\mathbf{K})\right|^{2}=\left|t_{12}^{s}\right|^{2}+\frac{\left|t_{12}^{p}\right|^{2}}{\left|n_{1}\right|^{2}\left|n_{2}\right|^{2} k_{0}^{4}}\left(\left|\gamma_{1}\right|^{2}+K^{2}\right)\left(\left|\gamma_{2}\right|^{2}+K^{2}\right) .
$$

Therefore,

$$
\begin{aligned}
& \left\langle E_{j}^{\mathrm{inc} *}(\mathbf{r}) E_{j}^{\mathrm{inc}}(\mathbf{r})\right\rangle \\
& =\frac{k_{0}^{4} \pi}{4 \varepsilon_{0}^{2}} \int^{(P)}(\omega) \int_{K=0}^{K=+\infty} \frac{1}{\left|\gamma_{1}\right|^{2}} e^{-\frac{(K \sigma)^{2}}{2}} \\
& \quad \times\left[\left|t_{12}^{s}\right|^{2}+\frac{\left|t_{12}^{p}\right|^{2}}{\left|n_{1}\right|^{2}\left|n_{2}\right|^{2} k_{0}^{4}}\left(\left|\gamma_{1}\right|^{2}+K^{2}\right)\left(\left|\gamma_{2}\right|^{2}+K^{2}\right)\right] \\
& \quad \times e^{-2 z \operatorname{Im} \gamma_{2}} \frac{1}{\operatorname{Im} \gamma_{1}} e^{-\frac{1}{2} \sigma^{2} \operatorname{Re} \gamma_{1}^{2}} K d K .
\end{aligned}
$$

Notice that since the conservative part of the electric force is $[28,33]\left\langle F_{i}^{e, \text { cons }}\right\rangle=\varepsilon_{0} \operatorname{Re} \alpha_{e} \partial_{i}\left\langle E_{j}^{\text {inc, } *}(\mathbf{r}) E_{j}^{\text {inc }}(\mathbf{r})\right\rangle / 4$ and the above expressions for the electric correlation tensors only depend on $z$, the gradient force components along $O X$ and $O Y$ are zero. The same arguments apply to the magnetic and interference forces.

In a similar way, the term proportional to $\operatorname{Im}\left\{\alpha_{e}\right\} \operatorname{Im}\left\{\left\langle E_{j}^{*}(\mathbf{r}) \partial_{i} E_{j}(\mathbf{r})\right\rangle\right\}$ of the nonconservative electric force $F_{i}^{e-\mathrm{nc}}$ is given by

$$
\begin{aligned}
\operatorname{Im}\{\langle & \left.\left\langle E_{j}^{*}(\mathbf{r}) \partial_{i} E_{j}(\mathbf{r})\right\rangle\right\} \\
= & \frac{k_{0}^{4}}{8 \varepsilon_{0}^{2}} \int^{(P)}(\omega) \operatorname{Im} \int_{0}^{2 \pi} d \theta \\
& \times \int_{0}^{\infty} i\left(K \cos \theta, K \sin \theta, \gamma_{2}\right) \frac{1}{\left|\gamma_{1}\right|^{2}} e^{-\frac{(K \sigma)^{2}}{2}} \\
& \times\left[\left|t_{12}^{s}\right|^{2}+\frac{\left|t_{12}^{p}\right|^{2}}{\left|n_{1}\right|^{2}\left|n_{2}\right|^{2} k_{0}^{4}}\left(\left|\gamma_{1}\right|^{2}+K^{2}\right)\left(\left|\gamma_{2}\right|^{2}+K^{2}\right)\right] \\
& \times e^{-2 z \operatorname{Im} \gamma_{2}} \frac{1}{\operatorname{Im} \gamma_{1}} e^{-\frac{1}{2} \sigma^{2} \operatorname{Re} \gamma_{1}^{2}} K d K .
\end{aligned}
$$

Only the $z$ component of this expression remains nonzero after performing the $\theta$ integration.

\section{APPENDIX B: GREEN'S FUNCTION $G_{i j}^{E p}$ AND $G_{i j}^{H m \leftrightarrow}$}

We now address the Green's function that accounts for the properties of the source through the reflection Fresnel coefficients $\left(r_{s, p}\right)[37,38]$

$$
G_{i j}^{E p}\left(\mathbf{r}, \mathbf{r}^{\prime}, \omega\right)=\frac{i}{2} \int_{-\infty}^{\infty} \frac{d^{2} \mathbf{K}}{(2 \pi)^{2}} G_{i j}^{E p}(\mathbf{K}) e^{i \mathbf{K}\left(\mathbf{R}-\mathbf{R}^{\prime}\right)} e^{i \gamma_{2}\left(z+z^{\prime}\right)},
$$

$$
\begin{aligned}
G_{i j}^{H m \leftrightarrow}\left(\mathbf{r}, \mathbf{r}^{\prime}, \omega\right)= & \frac{k_{0} n_{2}}{2} \int_{-\infty}^{\infty} \frac{d^{2} \mathbf{K}}{(2 \pi)^{2}} G_{i j}^{H m \leftrightarrow} \\
& \times(\mathbf{K}) e^{i \mathbf{K}\left(\mathbf{R}-\mathbf{R}^{\prime}\right)} e^{i \gamma_{2}\left(z+z^{\prime}\right)},
\end{aligned}
$$

where $G_{i j}^{E p}(\mathbf{K})=\frac{1}{\gamma_{2}}\left(\hat{s}_{i} r_{21}^{s} \hat{s}_{j}+\hat{p}_{1 i}^{+} r_{21}^{p} \hat{p}_{1 j}^{-}\right)$and $G_{i j}^{H m \leftrightarrow}(\mathbf{K})=$ $\frac{1}{\gamma_{2}}\left(\hat{p}_{1 i}^{+} r_{21}^{p} \hat{s}_{j}-\hat{s}_{i} r_{21}^{s} \hat{p}_{1 j}^{-}\right)$. The superindices $s$ and $p$ denote the Fresnel reflection coefficient $r_{21}$ at the plane $z=0$ on the side of region 2 under $s$ and $p$ polarization, respectively. Although four Green's functions are needed in order to calculate the electric (magnetic) field produced by the electric (magnetic) dipole, we only address the Green's function for the electric field produced by the electric and magnetic dipoles [cf. Eqs. (7) and (8), respectively], the rest of them are calculated from Maxwell's equations. Notice that in the Green's function described above there is no free-space term. This is due to the multiple scattering of the dipole field with the source surface. In this way, $\left\langle E_{i}^{p *}(\mathbf{r}) E_{i}^{p}(\mathbf{r})\right\rangle$ and $\left\langle E_{i}^{m *}(\mathbf{r}) E_{i}^{m}(\mathbf{r})\right\rangle$ are derived to obtain the different contributions to the force. This is done by using the above Green's functions and the procedure described in Appendix A.

In a similar way as before, only the $z$-component of the force becomes different from zero.
[1] L. Mandel and E. Wolf, Optical Coherence and Quantum Optics (Cambridge University Press, Cambridge, UK, 1995).

[2] T. H. Boyer, Phys. Rev. 174, 1764 (1968).

[3] T. H. Boyer, Phys. Rev. A 9, 2078 (1974).

[4] C. Henkel, K. Joulain, J.-P. Mulet, and J.-J. Greffet, J. Opt. A: Pure Appl. Opt. 4, S109 (2002).

[5] M. Antezza, L. P. Pitaevskii, and S. Stringari, Phys. Rev. Lett. 95, 113202 (2005).
[6] S. Y. Buhmann and S. Scheel, Phys. Rev. Lett. 100, 253201 (2008)

[7] A. W. Rodriguez, D. Woolf, A. P. McCauley, F. Capasso, J. D. Joannopoulos, and S. G. Johnson, Phys. Rev. Lett. 105, 060401 (2010); A. Madrazo, M. Nieto-Vesperinas, and N. Garcia, Phys. Rev. B 53, 3654 (1996); A. Garcia-Martin, J. A. Torres, J. J. Saenz, and M. Nieto-Vesperinas, Phys. Rev. Lett. 80, 4165 (1998). 
[8] D. Dalvit, P. Milonni, D. Roberts, and F. da Rosa, Casimir Physics, Lecture Notes in Physics (Springer, New York, 2011).

[9] A. W. Rodriguez, D. Woolf, P.-C. Hui, E. Iwase, A. P. McCauley, F. Capasso, M. Loncar, and S. G. Johnson, Appl. Phys. Lett. 98, 194105 (2011).

[10] K. Pan, A. P. McCauley, A. W. Rodriguez, M. T. H. Reid, J. K. White, and S. G. Johnson, Phys. Rev. A 83, 040503 (2011).

[11] J. Kohoutek, D. Dey, A. Bonakdar, R. Gelfand, A. Sklar, O. G. Memis, and H. Mohseni, Nano Lett. 11, 3378 (2011).

[12] K. Wang, E. Schonbrun, P. Steinvurzel, and K. B. Crozier, Nat. Commun. 2, 469 (2011).

[13] M. T. H. Reid, J. White, and S. G. Johnson, Phys. Rev. A 84, 010503(R) (2011).

[14] A. W. Rodriguez, D. Woolf, P.-C. Hui, E. Iwase, A. P. McCauley, F. Capasso, M. Lončar, and S. G. Johnson, Appl. Phys. Lett. 98, 194105 (2011).

[15] J. Varela, A. W. Rodriguez, A. P. McCauley, and S. G. Johnson, Phys. Rev. A 83, 042516 (2011).

[16] A. W. Rodriguez, F. Capasso, and S. G. Johnson, Nat. Photon. 5, 211 (2011), invited review.

[17] J. Zou, Z. Marcet, A. W. Rodriguez, M. T. H. Reid, A. P. McCauley, I. I. Kravchenko, T. Lu, Y. Bao, S. G. Johnson, and H. B. Chan, Nat. Commun. 4, 1845 (2013).

[18] A. B. Evlyukhin, C. Reinhardt, A. Seidel, B. S. Luk'yanchuk, and B. N. Chichkov, Phys. Rev. B 82, 045404 (2010).

[19] A. García-Etxarri, R. Gómez-Medina, L. S. Froufe-Pérez, C. López, L. Chantada, F. Scheffold, J. Aizpurua, M. NietoVesperinas, and J. J. Sáenz, Opt. Express 19, 4815 (2011).

[20] W. Liu, A. E. Miroshnichenko, D. N. Neshev, and Y. S. Kivshar, ACS Nano 6, 5489 (2012).

[21] J. A. Schuller, E. S. Barnard, W. Cai, Y. C. Jun, J. S. White, and M. L. Brongersma, Nat. Mater. 9, 193 (2010).

[22] B. Rolly, B. Stout, and N. Bonod, Opt. Express 20, 20376 (2012).

[23] J. A. Schuller, R. Zia, T. Taubner, and M. L. Brongersma, Phys. Rev. Lett. 99, 107401 (2007); M. Nieto-Vesperinas and E. Wolf, J. Opt. Soc. Am. A 2, 1429 (1985); M. Nieto-Vesperinas, ibid. 21, 491 (2004).

[24] M. Kerker, D.-S. Wang, and C. L. Giles, J. Opt. Soc. Am. 73, 765 (1983).

[25] A. I. Kuznetsov, A. E. Miroshnichenko, Y. H. Fu, J. Zhang, and B. Luk'yanchuk, Sci. Rep. 2, 57 (2012).

[26] A. B. Evlyukhin, S. M. Novikov, U. Zywietz, R. L. Eriksen, C. Reinhardt, S. I. Bozhevolnyi, and B. N. Chichkov, Nano Lett. 12, 3749 (2012).
[27] X. Fan, Z. Shen, and B. Luk'yanchuk, Opt. Express 18, 24868 (2010).

[28] J. M. Auñón and M. Nieto-Vesperinas, J. Opt. Soc. Am. A 29 , 1389 (2012).

[29] E. Lifshitz, Sov. Phys. JETP 2, 73 (1956).

[30] H. B. G. Casimir and D. Polder, Phys. Rev. 73, 360 (1948).

[31] J. W. Goodman, Statistical Optics, 1st ed. (John Wiley \& Sons, Inc., New York, 1985).

[32] M. Nieto-Vesperinas, Scattering and Diffraction in Physical Optics (World Science, Singapore, 2006).

[33] M. Nieto-Vesperinas, J. J. Sáenz, R. Gómez-Medina, and L. Chantada, Opt. Express 18, 11428 (2010).

[34] J. M. Auñón and M. Nieto-Vesperinas, Phys. Rev. A 85, 053828 (2012).

[35] J. D. Jackson, Classical Electrodynamics (Wiley, New York, 1998).

[36] J. M. Wylie and J. E. Sipe, Phys. Rev. A 30, 1185 (1984).

[37] J. E. Sipe, J. Opt. Soc. Am. B 4, 481 (1987).

[38] K. Joulain, J.-P. Mulet, F. Marquier, R. Carminati, and J.-J. Greffet, Surf. Sci. Rep. 57, 59 (2005).

[39] S. M. Rytov, Y. A. Kravtsov, and V. I. Tatarskii, Principles of Statistical Radiophysics. Part 3: Elements of Random Fields (Springer-Verlag, Berlin, 1989).

[40] M. Nieto-Vesperinas, R. Gomez-Medina, and J. J. Sáenz, J. Opt. Soc. Am. A 28, 54 (2011).

[41] J. Geffrin, B. García-Cámara, R. Gómez-Medina, P. Albella, L. Froufe-Pérez, C. Eyraud, A. Litman, R. Vaillon, F. González, M. Nieto-Vesperinas, J. Sáenz, and F. Moreno, Nat. Commun. 3, 1171 (2012).

[42] Y. H. Fu, A. I. Kuznetsov, A. E. Miroshnichenko, Y. F. Yu, and B. Luk'yanchuk, Nat. Commun. 4, 1527 (2013).

[43] M. Born and E. Wolf, Principles of Optics: Electromagnetic Theory of Propagation, Interference and Diffraction of Light (Cambridge University Press, Cambridge, UK, 1999).

[44] J. Chen, J. Ng, Z. Lin, and C. T. Chan, Nat. Photon. 5, 531 (2011).

[45] S. Sukhov and A. Dogariu, Phys. Rev. Lett. 107, 203602 (2011).

[46] A. Novitsky, C.-W. Qiu, and H. Wang, Phys. Rev. Lett. 107, 203601 (2011).

[47] T. Jouttenus, T. Setälä, M. Kaivola, and A. T. Friberg, Phys. Rev. E 72, 046611 (2005).

[48] S. Y. Buhmann and D.-G. Welsch, Prog. Quantum Electron. 31, 51 (2007).

[49] R. Carminati, Phys. Rev. A 81, 053804 (2010).

[50] C. Henkel, S. Ptting, and M. Wilkens, Appl. Phys. B 69, 379 (1999). 